International Atomic Energy Agency

and

United Nations Educational Scientific and Cultural Organization

INTERNATIONAL CENTRE FOR THEORETICAL PHYSICS

\title{
RELEVANCE OF INDUCED GAUGE INTERACTIONS IN DECOHERENCE
}

\author{
Dhurjati Prasad Datta円 \\ International Centre for Theoretical Physics, Trieste, Italy.
}

\begin{abstract}
Decoherence in quantum cosmology is shown to occur naturally in the presence of induced geometric gauge interactions associated with particle production. A new "gauge"variant form of the semiclassical Einstein equations is also presented which makes the non-gravitating character of the vacuum polarization energy explicit.
\end{abstract}

MIRAMARE - TRIESTE

July 1994

\footnotetext{
${ }^{1}$ Permanent Address: Department of Mathematics, North Eastern Regional Institute of Science and Technology, Itanagar 791109, India.
} 
Understanding the precise physical basis of a semiclassical regime of an interacting quantum system is of great current interest [1]. A particular aim of the recent studies is to find a mechanism [2,3] which should yield an almost classical observable from a given (nonclassical) dynamical system. These have a special relevance also in quantum cosmology (QC). Recently, there has been some attempts [4-11] in studying the origin and validity of the semiclassical Einstein equations (SCEE) in the framework of the quantum general relativity theory. The problem of realizing a classical observable universe from a possible quantum regime is also being addressed [5-7, 12] in the framework of the so called "decoherence process" in the quantum measurement theory $[2,3]$.

In Ref. [8-10] the problem of obtaining a correct set of back reacted SCEE is discussed in terms of the induced geometric gauge interactions. Apart from yielding clarifications to some longstanding ambiguities [13] in the semiclassical gravity, the study seems to offer new physical characterizations of the gravitational effects of the quantized matter fields.

It is well-known that geometric gauge interactions are induced spontaneously on a heavy dynamical system when it is acted upon by a light quantum system. In the language of the measurement theory, the light system may be considered as an environment. The natural framework of studying such an interaction is the Born-Oppenheimer (BO) approximation modified by the induced gauge fields [14]. The influence of the light system on the heavy one is succintly encoded in the form of the induced gauge fields, besides the usual BO potential term [15], once the light system is decoupled from the total system. The effects of these induced gauge fields are known to yield interesting physically testable predictions, e.g., the shift in energy spectra of the heavy system [14].

In semiclassical cosmology, the effects of the induced gauge fields seem to have deeper implications. One of the most important difference between the ordinary quantum mechanics and the QC is that in $\mathrm{QC}$ the universe is a closed system without a given a priori time. It is argued that the concept of time in cosmology may be an approximate one, retrieved possibly at a semiclassical regime. In such a case one should be able to separate from among the total infinite number of degrees of freedom, a smaller number of heavy degrees of freedom which behave quasiclassically in the environment of the remaining lighter degrees of freedom (matter + graviton). Although this separation seems to be realizable in the context of a minisuperspace, the physical implications of the same turn out [10] to be nontrivial.

The definition of the semiclassical time is found to be intimately related to the induced gauge structure, which in turn restricts severely the character of the BO potential in the effective Hamiltonian of the heavy degrees of freedom. In fact, it is shown [10] that the BO potential in $\mathrm{QC}$ is determined by the induced (vector) gauge connections so that the entire influence of the environment is encoded in the induced gauge fields. Physically, the environment leaves an imprint on the massive gravitational degrees of freedom in the form of an induced gauge bundle, which is nontrivial only if the gravitational minisuperspace has a nontrivial geometric/topological structure. In a (Lorentzian) Robertson-Walker (RW) minisuperspace e.g., the gauge bundle is trivial letting the associated gauge connection and hence the BO potential (which is actually an energy expectation value/transition matrix element) gauge equivalent to zero. An implication of this result is that the vacuum energy in a RW universe must be non-gravitating. Although counter-intuitive, this seems to offer a natural resolution of the problem why the cosmological constant in the present universe is negligibly small.

We note that the non-gravitating nature of the vacuum energy is a consequence of the 
semiclassical definition of time as a parametric derivative [6-11] which in turn relates it to the existence of a geometric phase [8-10]. When the phase is zero (the RW case), time cannot be defined at this level, reducing the (global) vacuum polarization energy nongravitating [10]. An interesting way of understanding this effect is through the analogy of the ordinary electromagnetism. The global vacuum energy carries an induced "magnetic" charge which does not contribute in the first (gravitational) energy integral. An immediate problem is then to suggest a new definition of time. This is however achieved by the next lower order "electric" type back reaction of the particles created by the time varying gravitational background. The particle production effect is associated with a nontrivial Pancharatnam phase [9] which is pinned by an Euclidean time integral of the total decay width of the unstable state. In Ref. [10] we obtain the electric type potential from the particle production effect and its back reaction in SCEE. The physical time is then shown to have its origin from this Euclidean time by an analytic continuation. Below we give further justification of this construction by expressing the SCEE in a suitably defined new "gauge". However, one still needs to clarify in more detail the exact meaning of the "initial" Euclidean time. A pertinent question is: what was, if any, before the Euclidean era?

Further as stated already the origin of time needs the existence of a nontrivial geometric phase which in turn needs to single out a unicomponent complex WKB state in the gravitational sector $[10,11]$. One should therefore also clarify the relevance of the Berry geometric phase in the context of decoherence, which we do here.

The relevance of decoherence and correlations in QC in connection with the back reacted SCEE is being studied by $\mathrm{Hu}$ and collaborators [4-7] for sometime. Paz and Sinha [6] in particular have shown that the SCEE can be derived by demanding decoherence in different WKB branches in the universe wave function. The importance of achieving correlations (in the sense of a gaussian peak in the Wigner function) between relevant physical degrees of freedom in a single WKB branch is also emphasized in the context of specific models. According to the decoherence paradigm [2] the "relevant" quantum degrees of freedom are constantly being measured continuously by the environment degrees of freedom through some nontrivial interaction. The environment degrees of freedom are however not observable. These degrees of freedom should therefore be traced out in the total density matrix of the system and environment. This leads to a reduced density matrix of the system which includes the influence of the environment on the system. The effects of environment on the system get encoded in the Feynmann-Vernon (FV) influence functional [16] which brings out the nonlocal character of the same. As noted in Ref. [6] the back reactions turns out to depend on the histories of the system variables. The relevance of particle production in decoherence is also pointed out $[5,6]$. Recently, the relation between this approach in QC and the quantum Brownian problem has been stressed. Some interesting relationships between dissipation; noise and the cosmological particle production have been proposed [7].

However, this approach still seems to be incomplete since the possibility of an induced gauge interaction in the Feynman-Vernon functional has not been explored. Here, we show that decoherence is actually controlled by the nontrivial geometric phase associated with particle production. A loss of quantum coherence in $\mathrm{QC}$ is thus related to the emergence of a semiclassical time. B Consider a minisuperspace model with $N$ degrees of freedom denoted by the coordinates $q_{i}(i=1, \ldots, N)$. The minisuperspace modes are coupled to the environment degrees of freedom that are denoted here by a scalar field 
$\varphi$. The quantum mechanical description of this interacting system is governed by the Wheeler-Dewitt (WD) equation:

$$
H \Psi \equiv\left(H_{g}+H_{m}\right) \Psi(q, \varphi)=0
$$

Here $\Psi(q, \varphi)$ is the total wavefunction of the universe filled with matter $\varphi$. For definiteness, we consider the gravitational Hamiltonian $\mathrm{Hg}$ in the form

$$
H g=\frac{1}{2 M} G^{i j} P_{i} P_{j}+M V(q)
$$

$G^{i j}$ denotes the metric in the minisuperspace, $P_{i}$ the momentum conjugate to the configuration variables $q_{i}, V(q)$ the superpotential. The quantity $M$ is proportional to the square of the Planck mass. The Hamiltonian for the matter fields (environment) $H_{m}(\varphi, q)$ is kept arbitrary. Because of the large value of $M$ compared to the ordinary matter mass scales, one can use BO approximation to discuss the solutions of (1).

To apply the improved BO approximation to Eq.(1) we write

$$
\Psi=\psi(q) \chi(q, \varphi)
$$

and assume that the quasiclassical gravitation modes $q$ are adiabatic [17]. Then integrating the total Hamiltonian between an initial and a final matter states $\chi_{i}$ and $\chi_{f}$ respectively, one gets the effective gravitational WD equation

$$
H_{e f f} \psi(q) \equiv\left[\frac{1}{2 M} G^{i j}\left(P_{i}-\hbar A_{i}\right)\left(P_{j}-\hbar A_{j}\right)+M V+\frac{\left\langle\chi_{f}\left|H_{m}\right| \chi_{i}\right\rangle}{\left\langle\chi_{f} \mid \chi_{i}\right\rangle}+\frac{\hbar}{2 M} \rho\right] \psi=0
$$

where the induced $U(1)$ gauge connection $A_{i}$ and the electric type potential $\rho$ is given by $[10,15]$

$$
\begin{gathered}
A_{i}=i \frac{\left\langle\chi_{i} \mid \partial_{i} \chi_{i}\right\rangle}{\left\langle\chi_{i} \mid \chi_{i}\right\rangle}, \quad \partial_{i}=\frac{\partial}{\partial q_{i}} \\
\rho=\hbar G^{i j} \frac{\left\langle\chi_{f}\left|\left(P_{i}-\hbar A_{i}\right)\left(P_{j}-\hbar A_{j}\right)\right| \chi_{i}\right\rangle}{\left\langle\chi_{f} \mid \chi_{i}\right\rangle}
\end{gathered}
$$

For details of the derivation of Eq. (4) we refer to Ref. [9].

Now a semiclassical regime is assumed to be one when the effective gravitational wave function $\psi(q)$ can be approximated by an oscillatory WKB state $\psi \sim \exp \left(\frac{i}{\hbar} S(q)\right)$. In this case the matter state $\chi(q, \varphi)$ corresponds to a curved space Schrödinger wave functional

$$
i \hbar \frac{d}{d t}|\chi\rangle=H_{m}|\chi\rangle
$$

where the semiclassical WKB time is defined by

$$
\frac{d}{d t}=G^{i j} \frac{\partial S}{\partial q_{i}} \frac{\partial}{\partial q_{j}} \Rightarrow \frac{d q i}{d t}=G^{i j} \frac{\partial S}{\partial q_{j}}=G^{i j} \frac{P_{j}}{M}
$$

At a particular value of the superspace variable $q,|\chi\rangle$ thus stands for a suitable Fock state constructed using the formalism of the curved space quantum field theory. The choice of a vacuum, e.g., cannot therefore be made unambiguously. However, under the assumed adiabatic condition, one may be able to pick an adiabatic vacuum [18] which we do here. 
For a general case special symmetry (e.g., de Sitter or conformal) may need to be invoked. The choice of an adiabatic vacuum is however sufficient for our purpose of showing the relevance of geometric phase in decoherence.

The SCEE with back reactions now can be written as,

$$
\frac{1}{2 M} G^{i j}\left(P_{i}-\hbar A_{i}\right)\left(P_{j}-\hbar A_{j}\right)+M V+\frac{\left\langle\chi_{f}\left|H_{m}\right| \chi_{i}\right\rangle}{\left\langle\chi_{f} \mid \chi_{i}\right\rangle}+\frac{\hbar}{2 M} \rho=0
$$

where the classical effective momentum $P_{i}$ is defined by [c.f. Eq. (7)]

$$
P_{i}=M \frac{\partial S}{\partial q_{i}}
$$

We note that the transition matrix element $\left\langle H_{m}\right\rangle=\left\langle\chi_{f}\left|H_{m}\right| \chi_{i}\right\rangle /\left\langle\chi_{f} \mid \chi_{i}\right\rangle$ (expectation values in case $\left.\left|\chi_{f}\right\rangle \equiv\left|\chi_{i}\right\rangle\right)$ carries a geometric "magnetic" charge:

$$
\left\langle H_{m}\right\rangle=\hbar A_{i} \frac{d q_{i}}{d t}
$$

which follows from Eqs. (6) and (7). The last lower order term $\rho$ in Eq. (8), however, corresponds to an "electric" potential [15] for a particle (in minisuperspace) with an "electric" charge $\hbar / 2 M$. This also gives the back reaction for the particles created on the background geometry. The effect of renormalization on the vacuum transition element $\left\langle H_{m}\right\rangle$ has been discussed in Ref. [19]. The local geometrical part of the vacuum polarization contributes to the higher derivative corrections in the Einstein-Hilbert action and has well defined gravitational effect. (For simplicity we fix the renormalized higher derivative coupling constants to zero). However, for a flat simply connected minisuperspace (e.g. the RW and most of the homogeneous models) the induced $U(1)$ bundle is trivial. Thus one can always make a gauge choice reducing the global renormalized vacuum polarization component of $\left\langle H_{m}\right\rangle$ to zero.

However, the adiabatic variation of the background geometry is expected also to produce nonperturbative effects in the matter sector. This induces exponentially small imaginary part in the transition matrix element: $\operatorname{Im}\left\langle H_{m}\right\rangle=\Gamma$, the total decay width of the vacuum. This lack of adiabaticity can however be treated in the adiabatic perturbation theory using the Euclidean time formalism [9]. The Euclidean integral

$$
\gamma_{P}=\hbar^{-1} \int_{i}^{f} \Gamma d \tau, \quad \tau=i t
$$

gives the nontrivial geometric Pancharatnam phase between the initial and final vacua. In fact, the potential $\hbar \rho / 2 M$ is an effect of this nonintegrable phase in the SCEE. The SCEE derived from a simply-connected flat minisuperspace thus assumes the form [10]

$$
\frac{1}{2 M} G^{i j} P_{i} P_{j}+M V+\frac{\hbar}{2 M} \rho=0
$$

A remark on the definition of time is in order here.

The vacuum energy $E_{0}=R e\left\langle H_{m}\right\rangle$ being gauge equivalent to zero, a physical time $t$ cannot be immediately written down via Eq. (7). The WKB time should originally be retrieved as an Euclidean parameter

$$
\frac{d}{d \tau}=G^{i j} \frac{\partial S^{E}}{\partial q_{i}} \frac{\partial}{\partial q_{j}}
$$


where the Euclidean action $S^{E}=i S$. A simple way of recovering the physical time $t$ is now to make a "gauge" transformation

$$
\frac{d}{d \tilde{\tau}}=e^{\hbar^{-1} \int E_{0} d \tau} \frac{d}{d \tau} e^{-h^{-1} \int E_{0} d \tau}
$$

and then introduce the rotation $\tilde{\tau}=i \tilde{t}$. The Schrodinger Eq. (6) then assumes the form

$$
i \hbar \frac{d}{d \tilde{t}}|\chi\rangle=\tilde{H}_{m}|\chi\rangle, \quad \tilde{H}_{m}=H_{m}-E_{0}
$$

The time derivative entering in momentum $P_{i}$ in Eq. (12) should also be taken with respect to $\tilde{t}$ (this amounts to a gauge transformation eluded already). Eqs. (13)-(15) and (12) now constitute the final form of the SCEE and the matter Schrodinger equation. The virtue of this "gauge"-transformed representation is that it makes manifest the nongravitating nature of the vacuum energy. (We omit tilde henceforth). The transformation (14) seems to eat up the apparent non-zero vacuum polarization energy in Eq.(6).

We note that the solution $\psi(q)$ of the effective WD equation (4) represents a class of $(N-1)$-parameter solutions $\psi_{n}(q)[6]$. The total wave function should therefore be written as a general superposition of the form

$$
\Psi(q, \varphi)=\Sigma \psi_{n}(q) \chi_{n}(q, \varphi)
$$

The definition of time (7) and hence the matter Schrodinger state $\chi_{n}$ thus depends on the specific choice of the WKB branch $\psi_{n} \sim \exp \left(i S_{n} / \hbar\right)$. It is therefore desirable to justify the choice of a single component WKB state $\psi_{n}$ for a semiclassical description of the Universe. This will be achieved by showing that decoherence occurs both between different WKB branches and inside a single WKB component. In fact, the appearance of a nontrivial geometric phase in connection with particle production along each WKB branch seems to destroy the quantum coherence of the total quantum system.

As discussed by Kiefer [12] and Paz and Sinha [6] the problem of decoherence in QC can also be addressed in terms of the FV influence functional [16]. This is defined by introducing the reduced density matrix of the universe

$$
\rho_{r}\left(q, q^{\prime}\right)=\int d \varphi \Psi^{*}(q, \varphi) \Psi\left(q^{\prime}, \varphi\right)
$$

when all the irrelevant environment degrees of freedom are integrated out. Using Eq. (16), the reduced density matrix can be rewritten as:

$$
\rho_{r}\left(q, q^{\prime}\right)=\sum_{n . n^{\prime}} \psi_{n}^{*}(q) \psi_{n^{\prime}}\left(q^{\prime}\right) F_{n n^{\prime}}\left(q, q^{\prime}\right)
$$

where the FV influence functional is given by [12]

$$
F_{n n^{\prime}}\left(q, q^{\prime}\right)=\int d \varphi \chi_{n}^{*}(q, \varphi) \chi_{n^{\prime}}\left(q^{\prime}, \varphi\right)
$$

Note that the influence functional is in general a nonlocal functional of histories $q(t)$ and $q^{\prime}\left(t^{\prime}\right)$ of the semiclassical universes as predicted by the WKB branches $\psi_{n}(q)$ and $\psi_{n^{\prime}}\left(q^{\prime}\right)$ respectively. For the same WKB branch $n=n^{\prime}$, the different histories $q$ and $q^{\prime}$ correspond to interference between a possible expanding and collapsing mode. For decoherence to 
occur both the off-diagonal terms $F_{n n^{\prime}}(q, q)$ and $F_{n n}\left(q, q^{\prime}\right)$ in the reduced density matrix should be exponentially suppressed. The reduced density matrix $\rho_{r}$ will then be a sum of non-interfering WKB branches indicating the onset of a classical era.

The close similarity of the integral (19) and the close-time-path vacuum generating functional has been noted by Calzetta and $\mathrm{Hu}$ [20] recently. The integral (19) in fact gives the transition of an in-vacuum, prepared at a fictitious time $t=-\infty$, along the history $q$ upto $t=T$ and then return to the in-vacuum again at $t=-\infty$ along $q^{\prime}$. The matter field histories $\phi$ and $\phi^{\prime}$ are supposed to match at time $T: \phi(T)=\phi^{\prime}(T)$. Let the common in vacuum be $\left|\chi_{0}\right\rangle$. Then one has

$$
F_{n n^{\prime}}\left(q, q^{\prime}\right)=\left\langle\chi_{0} \mid \chi_{n}^{\prime}\right\rangle_{q^{\prime}}\left\langle\chi_{n} \mid \chi_{0}\right\rangle_{q}
$$

To choose an adiabatic in-vacuum we need to invoke a possible switching off mechanism of the gravitational interaction at $t \rightarrow-\infty$ [6]. It is well known [6] that this introduces restrictions on the class of admissible super potentials $V(q)$. We note that relative to the in-vacuum $\left|\chi_{0}\right\rangle$, the state $\left|\chi_{n}\right\rangle$ will in general be a multiparticle state in the out region $T \rightarrow \infty$. The amplitude $\left\langle\chi_{0} \mid \chi_{n}\right\rangle_{q}$ then gives a measure of the total shift in the in-vacuum due to the adiabatic evolution along the history $q$. To evaluate the amplitude we use the adiabatic theorem [14]:

$$
\ln \left\langle\chi_{0} \mid \chi_{n}\right\rangle_{q}=\ln \left(\frac{1}{\left|1+i \beta_{n}\right|}\right)-i \gamma_{n}-i \frac{\hbar}{2 M} \int \rho_{n} d t
$$

where the lowest order phase correction $\gamma_{n}$ corresponds to the geometric phase

$$
\gamma_{n}=\hbar^{-1} \int E_{n} d t+\hbar^{-1} \int \Gamma_{n} d \tau
$$

Here, the state $\left|\chi_{n}\right\rangle$ is assumed to be normalized throughout the entire period of its evolution. Further, $E_{n}$ denotes the instantaneous adiabatic energy, $\Gamma_{n}$ the decay width of the state [9] and $\rho_{n}$ an higher order correction to the geometric phase [14]. The exponential decay of the state $\left|\chi_{n}\right\rangle$ is determined by $\beta_{n}$ which is in general related to the decay width $\Gamma_{n}$ and the energies associated to the states $\left|\chi_{0}\right\rangle$ and $\left|\chi_{n}\right\rangle$.

The final form of the influence functional can now be written as

$$
\begin{aligned}
F_{n n^{\prime}}\left(q, q^{\prime}\right)= & \exp \left[i \left\{\hbar^{-1}\left(\int E_{n} d t-\int E_{n^{\prime}} d t^{\prime}\right)+\hbar^{-1}\left(\int \Gamma_{n} d t-\int \Gamma_{n^{\prime}} d t^{\prime}\right)\right.\right. \\
& \left.\left.+\frac{\hbar}{2 M}\left(\int \rho_{n} d t-\int \rho_{n^{\prime}} d t^{\prime}\right)\right\}\right] \times \exp \left[-\left(\beta_{n}-\beta_{n^{\prime}}\right)^{2}\right]
\end{aligned}
$$

In view of the fact that $\beta_{n} \propto \Gamma_{n}$ in the adiabatic approximation, one concludes that the expected decoherence is obtained whenever there is a large difference in geometric phases (associated with particle production) along the two histories. Further, the geometric phases associated with distinct histories ought to be different. Otherwise the histories will be homotopically equivalent, which follows from the topological invariance of the geometric phase. This phase difference indicates varying rates of particle production along different WKB histories, suppressing the off-diagonal terms. Stated in other words, the loss of quantum coherence in WKB branches can be seen as due to the lack of coherence in the induced geometric phases. This seems to be a new interpretation of the relationship 
between decoherence and cosmological particle production noted already by many authors $[5-7]$.

In the case of a RW universe, the energy integrals in Eq.(23) can be gauged away [WKB time $t\left(t^{\prime}\right)$ in this case is identical to one in Eq. (15)]. The simplified influence functional then has the form

$$
\begin{aligned}
F_{n n^{\prime}}\left(q, q^{\prime}\right)=\exp \left[i \left\{\hbar ^ { - 1 } \left(\int\right.\right.\right. & \left.\left.\left.\Gamma_{n} d \tau-\int \Gamma_{n^{\prime}} d \tau^{\prime}\right)+\frac{\hbar}{2 M}\left(\int \rho_{n} d t-\int \rho_{n^{\prime}} d t^{\prime}\right)\right]\right] \\
& \times \exp \left(-\left(\beta_{n}-\beta_{n^{\prime}}\right)^{2}\right)
\end{aligned}
$$

One notes the interesting functional relationship between the phase and the damping factor of the influence functional. The explicit derivation of the cosmological fluctuationdissipation theorem [7] in the present context will be taken up separately.

Finally, as an application let us compute the influence functional damping factor in a toy model. The model consists of a RW minisuperspace coupled to a conformal matter field $[5,6]$. Since detailed calculations are available in literature we will be brief bringing out the main features of the present approach.

The relevant WD equation has the form

$$
\left[\frac{1}{2 M} \frac{\partial^{2}}{\partial a^{2}}+M V(a)+\frac{1}{2} \sum_{k}\left(-\frac{\partial^{2}}{\partial \varphi_{k}^{2}}+\Omega_{k}^{2}(a) \varphi_{n}^{2}\right)\right] \psi=0
$$

Here, $\varphi_{k}$ are the scalar field eigenmodes of the spatial Laplacian $\Delta$ on the unit 3 -sphere, $\Omega_{k}^{2}=k^{2}+m^{2} a^{2}, m$ the mass of the scalar field and $a$ the RW scale factor. The matter wave function $\chi(a, \varphi)$ turns out to be seperable

$$
\chi(a, \varphi)=\prod_{k} \chi_{k}\left(a, \varphi_{k}\right)
$$

where each component $\chi_{k}$ satisfies the Schrodinger equation

$$
i \hbar \frac{d}{d t} \chi_{k}=\left(-\frac{1}{2} \frac{\partial^{2}}{\partial \phi_{k}^{2}}+\Omega_{k}^{2} \varphi_{k}^{2}\right) \chi_{k}
$$

and $t$ is the WKB time defined by $\frac{d a}{d t}=\frac{d S}{d a}$. Let us assume a Gaussian ansatz for the wave function $\chi$ (we omit the index $k$ for simplicity)

$$
\chi(a, \varphi)=\left(\frac{\varepsilon}{\pi}\right)^{1 / 4} e^{i \alpha(a)-\frac{1}{2} \sigma(a) \varphi^{2}}
$$

where $\mathrm{v}$

$$
\begin{gathered}
\sigma=\varepsilon+i \Gamma \\
2 \hbar \dot{\alpha}=-\varepsilon \text { and } \hbar \dot{\sigma}=-i \sigma^{2}+i \Omega_{k}^{2}
\end{gathered}
$$

so that $\langle\chi \mid \chi\rangle=1$. The initial state $\left|\chi_{0}\right\rangle$ thus turns out to be the harmonic oscillator ground state with energy $\varepsilon_{0}=\frac{1}{2} \Omega(0) \hbar$. It is now straightforward to calculate

$$
\left\langle\chi_{0} \mid \chi\right\rangle=e^{-i \Gamma / 4 \varepsilon_{0}}\left|1+i \frac{\Gamma}{2 \varepsilon_{0}}\right|^{-1 / 2}
$$

where we use first order adiabatic correction in evaluating the wave function $|\chi\rangle[6]$. We also absorb the phase contribution from the energy integral in the definition of time (c.f. 
Eq. (15)). Note, however, the geometric phase $\Gamma / 4 \varepsilon_{0}$ associated to the decay of the state. The influence functional then assumes the form (inserting the index $k$ ) $\mathrm{v}$

$$
F\left(a, a^{\prime}\right)=\exp \left[i \Sigma \frac{1}{4 \varepsilon_{0 k}}\left(\Gamma_{k}-\Gamma_{k}^{\prime}\right)\right] \exp \left[-\Sigma \frac{1}{8 \varepsilon_{0 k}^{2}}\left(\Gamma_{k}-\Gamma_{k}^{\prime}\right)^{2}\right]
$$

This should be compared with the expression obtained by Paz and Sinha [6]. Although the damping factor is identical, the influence phase is different. One reason for the difference is the non-gravitating nature of the vacuum polarization energy.

To conclude, we have shown the close proximity of decoherence with the occurrence of the nontrivial geometric phase in an interacting system. In the cosmological context discussed here, decoherence of the WKB branches of the universe histories is realized provided there is a large uncertainty in the induced phase differences, reflecting a vastly different rates in particle production. However, our result is still not sufficiently general because of the adiabatic switching off mechanism used here. It is desirable to remove this restriction from the argument. Finally, we have also presented a new "induced gauge" variant form of the SCEE, rendering the non-gravitating character of the global renormalized vacuum energy manifest. These results seem to have interesting implications in quantum gravity and quantum measurement problems.

\section{Acknowledgments}

It is a pleasure to thank Professor S. Randjbar-Daemi, Professor A.O. Caldeira and Professor Deepak Kumar for fruitful discussions, Dr. L. Palacios for numerous help and constant encouragement, and Dr. C. Kiefer for a number of stimulating correspondence over electronic mail. The author would also like to thank Professor Abdus Salam, the International Atomic Energy Agency and UNESCO for hospitality at the International Centre for Theoretical Physics, Trieste. v 


\section{References}

[1] W.H. Zurek and J.P. Paz, Phys. Rev. Lett. 72, 2508 (1994); Chaos and Quantum Physics, Proceedings of the Les Houches Summer School, session LII (North Holland, Amsterdam, 1991).

[2] W.H. Zurek, Phys. Today 44, No. 10, 36 (1991), 46; No. 12, 81 (1993); Prog. Theor. Phys. 89, 281 (1993).

[3] M. Gell-Mann and J.B. Hartle, Phys. Rev. D47, 3345 (1993).

[4] E. Calzetta and B.L. Hu, Phys. Rev. D35, 495 (1987); 40, 656 (1989); B.L. Hu, Physica A158, 399 (1989); B.L. Hu and A. Matacz, "Back reaction in semiclassical cosmology: the Einstein-Langevin equation", University of Maryland Preprint umdpp 94-31.

[5] E. Calzetta and F. Mazzitelli, Phys. Rev. D42, 4066 (1990).

[6] J.P. Paz and S. Sinha, Phys. rev. D44, 1038 (1991); 45, 2823 (1992).

[7] B.L. Hu and S. Sinha, "A fluctuation-dissipation relation for semiclassical cosmology", university of Maryland preprint, umdpp 94-164.

[8] D.P. Datta, Mod. Phys. Lett. A8, 191 (1993); 8, 2523(E)(1993); 8, 601 (1993); "semiclassical back reaction from Wheeler-Dewitt equation", report (unpublished).

[9] D.P. Datta, Phys. Rev. D48, 5746 (1993).

[10] D.P. Datta, Phys. Rev. D49 (1994) 2605; "Can vacuum energy gravitate?" submitted for publication (1994).

[11] C. Kiefer in "Canonical gravity - from classical to quantum", ed. by J. Ehlers and H. Friedrich (Springer, Berlin, 1994).

[12] C. Kiefer, Class. Quantum Grav. 8, 379 (1991);

J.J. Halliwell, Phys. Rev. D39, 2912 (1989);

T. Padmanabhan, ibid. 39, 2924 (1989).

[13] J.J. Halliwell, Phys. Rev. D36, 3627 (1987);

T.P. Singh and T. Padmanabhan, Ann. Phys. (NY) 196, 296 (1989).

[14] Geometric phases in Physics, ed. by A. Shapere and F. Wilczek (World Scientific, Singapore, 1989).

[15] M.V. Berry and J.M. Robbins, Proc. R. Soc. Lond. A442, 641 (1993).

[16] R.P. Feynman and F.L. Vernon, Ann. Phys. (NY) 24, 118 (1963).

[17] We impose the condition of adiabaticity at the beginning. This is to be contrasted with the other approaches [6] where the consistency of the SCEE was conjectured only at an adiabatic limit. Moreover, predictions from the transition matrix elements and the expectation value seem to agree under the adiabatic condition. 
[18] N. Birrell and P.C.W. Davies, Quantum Fields in Curved Space (Cambridge University Press, Cambridge, 1982).

[19] D.P. Datta, "Geometric gauge fields in semiclassical gravity" ICTP, Internal Report, IC/94/146 (1994).

[20] E. Calzetta and B.L. Hu, Phys. Rev. D49, 6636 (1994). 